\title{
COUNTEREXAMPLE TO A THEOREM ON DIFFERENTIAL EQUATIONS IN HILBERT SPACE
}

\author{
WILLIAM J. KNIGHT
}

ABSTRACT. A counterexample is given to a theorem by Felix Browder concerning solutions of differential equations with values in Hilbert space. Several valid conclusions (as near the original conclusions as possible) are pointed out.

In 1964 Felix E. Browder [1, p. 518] published the following theorem: Let $H$ be a Hilbert space, $f$ a weakly continuous mapping of $R^{+} \times H$ into $H$. Then for each $r>0$, there exists $a(r)>0$ such that, for each $u_{0}$ in $H$ with $\left\|u_{0}\right\|<r$, there exists a strongly $C^{1}$-solution $u$ of

$$
d u(t) / d t=f(t, u(t)), \quad 0 \leq t<a(r),
$$

with $u(0)=u_{0}$.

This theorem has been cited more recently in [2] and [4]. However, the conclusion of the theorem is too strong, as we shall show by a counterexample. It is possible to conclude that the solution $u$ is absolutely continuous (in the sense of Hille and Phillips [3, p. 76]), strongly differentiable a.e. to $f(t, u(t))$ on $[0, a(r))$, and even weakly differentiable everywhere to $f(t, u(t))$ on $[0, a(r))$, but $u$ may fail to be (right) differentiable in the strong sense at 0 .

The proof given in [1] for the stated theorem constructs a function $u$ on $[0, a(r))$ satisfying

$$
\langle u(t), v\rangle=\left\langle u_{0}, v\right\rangle+\int_{0}^{t}\langle f(s, u(s)), v\rangle d s \quad \text { (Riemann integral) }
$$

for every $t$ in $[0, a(r)$ ) and every $v$ in $H$. (Here $\langle$,$\rangle denotes the inner prod-$ uct on $H_{\text {.) }}$ The equation above does not imply that

$$
u(t)=u_{0}+\int_{0}^{t} f(s, u(s)) d s \quad(H \text {-valued Riemann integral })
$$

Received by the editors April 16, 1974.

AMS (MOS) subject classifications (1970). Primary 35G05; Secondary 46G05.

Key words and phrases. Nonlinear differential equation, Hilbert space, strongly $C^{1}$-solution, weakly continuous map, weak derivative, Pettis integral, Bochner integral. 
for all $t$ in $[0, a(r))$. It implies only that

$$
\left.u(t)=u_{0}+\int_{0}^{t} f(s, u)\right) d s \quad \text { (Pettis integral) }
$$

for all $t$ in [0, $a(r))$. (See Hille and Phillips [3, p. 78].) However, this tells us that $u$ is absolutely continuous, whence $f(s, u(s))$ is bounded and strongly measurable if $0 \leq s \leq t<a(r)$. So in fact

$$
u(t)=u_{0}+\int_{0}^{t} f(s, u(s)) d s \quad \text { (Bochner integral) }
$$

for all $t$ in [0, $a(r))$. Thus $u(t)$ is strongly differentiable almost everywhere to $f(i, u(t))$ on $[0, a(r))$, and $u^{\prime}(t)$ is weakly continuous. Furthermore, $u(t)$ is weakly differentiable to $f(t, u(t))$ at every point $t$ in $[0, a(r))$, since for all $v$ in $H$ and all $t$ in $[0, a(r))$ we have

$$
\begin{aligned}
\lim _{h \rightarrow 0}\left\langle\frac{u(t+h)-u(t)}{h}, v\right\rangle & =\lim _{h \rightarrow 0}\left\langle\frac{1}{h} \int_{t}^{t+h} f(s, u(s)) d s, v\right\rangle \\
& =\lim _{h \rightarrow 0} \frac{1}{h} \int_{t}^{t+h}\langle f(s, u(s)), v\rangle d s=\langle f(t, u(t)), v\rangle
\end{aligned}
$$

by the fundamental theorem of calculus for Riemann integrals.

The counterexample. We construct a weakly continuous function $f:[0,1] \rightarrow l_{2}$ such that the function $F$ defined by $F(t)=\int_{0}^{t} f(s) d s$ (Bochner integral) is not strongly differentiable at 0 . Then the problem

$$
d u(t) / d t=f(t), \quad u(0)=0
$$

satisfies the hypotheses of Browder's theorem. However, if a strongly $C^{1}$. solution $u$ existed, it would then satisfy

$$
u(t)=\int_{0}^{t} f(s) d s=F(t) \quad \text { (Bochner integral) }
$$

for all small $t$, but this function fails to be strongly differentiable at $t=0$.

The function $f$ is defined as follows.

(a) For $n=1,2,3, \ldots$, we define scalar valued functions $g_{n}$ by

$$
g_{n}(t)=\left\{\begin{array}{l}
\left(2^{-n+1}-2^{-n}\right)^{-4}\left(2^{-n+1}-t\right)^{2}\left(t-2^{-n}\right)^{2} \text { if } t \in\left(2^{-n}, 2^{-n+1}\right), \\
0 \quad \text { otherwise }
\end{array}\right.
$$

for all $t$ in $[0,1]$.

(b) For $n=1,2,3, \ldots$, we set $f_{n}(t)=d\left[\operatorname{tg}_{n}(t)\right] / d t$ for all $t$ in $[0,1]$.

(c) The function from $[0,1]$ into $l_{2}$ is defined at $t$ to be the sequence 


$$
f(t)=\left\{f_{1}(t), f_{2}(t), f_{3}(t), \ldots\right\}
$$

Note that for each $t$ in $[0,1]$, at most one term of $f(t)$ is nonzero, and thus $f(t)$ certainly belongs to $l_{2}$ for each $t$ in $[0,1]$. This shows also that $\|f(t)\|_{2}=\left|f_{n}(t)\right|$ for the unique $n$ such that $t \in\left(2^{-n}, 2^{-n+1}\right]$.

Let $t_{n}^{*}$ denote the midpoint of $\left[2^{-n}, 2^{-n+1}\right]$. Then $g_{n}\left(t_{n}^{*}\right)=2^{-4}$, $g_{n}^{\prime}\left(t_{n}^{*}\right)=0$, and $f_{n}\left(t_{n}^{*}\right)=2^{-4}$ when $n=1,2,3, \ldots$ Thus strong $\lim _{t \rightarrow 0} f(t) \neq$ 0 , and so $f$ is not strongly continuous at 0 . However, it is easy to see that $f$ is strongly continuous everywhere on the half-open interval $(0,1]$ and weakly continuous even at 0 . (One shows that $\lim _{t \rightarrow 0} f_{n}(t)=0$ and $\|f(t)\|_{2}$ is bounded by 5 as $t \rightarrow 0$.)

Now consider the function $F$ defined by $F(t)=\int_{0}^{t} f(s) d s$ (Bochner integral) for $t$ in $[0,1]$. Clearly $F(t)=\left\{F_{1}(t), F_{2}(t), F_{3}(t), \ldots\right\}$ where $F_{n}(t)=$ $\int_{0}^{t} f_{n}(s) d s=\operatorname{tg}_{n}(t)$. Then

$$
\frac{F(t)-F(0)}{t-0}=t^{-1} F(t)=\left\{g_{1}(t), g_{2}(t), g_{3}(t), \ldots\right\}
$$

for all $t$ in $(0,1]$. Now each $g_{n}(t) \rightarrow 0$ as $t \rightarrow 0$, but

$$
\left\|\left(t_{n}^{*}\right)^{-1} F\left(t_{n}^{*}\right)\right\|_{2}=\left|g_{n}\left(t_{n}^{*}\right)\right|=2^{-4} \text { for all } n \text {. }
$$

Thus strong $\lim _{t \rightarrow 0} t^{-1} F(t)$ fails to exist, and so $F(t)$ is not strongly differentiable at $t=0$. This completes the counterexample.

If we wish to alter $f$ so as to make the solution of $u^{\prime}(t)=f(t)$ fail of strong differentiability át the points $t_{n}=1 / n, n=1,2,3, \ldots$, we need only string together copies of the function $f$ constructed above, but tailored to fit the intervals $[1 /(n+1), 1 / n]$.

\section{BIBLIOGRAPHY}

1. Felix E. Browder, Non-linear equations of evolution, Ann. of Math. (2) 80 (1964), 485-523. MR 30 \#4167.

2. J. B. Diaz and R. J. Weinacht, On nonlinear differential equations in Hilbert spaces, Applicable Anal. 1 (1971), no. 1, 31-41. MR 43 \#7746.

3. E. Hille and R. S. Phillips, Functional analysis and semi-groups, rev. ed., Amer. Math. Soc. Colloq. Publ., vol. 31, Amer. Math. Soc., Providence, R. I., 1957. MR 19, 664 .

4. L. A. Medeiros, On nonlinear differential equations in Hilbert spaces, Amer. Math. Monthly 76 (1969), 1024-1027. MR 40 \#1685.

DEPARTMENT OF MATHEMATICS, PENNSYLVANIA STATE UNIVERSITY, UNIVERSITY PARK, PENNSYLVANIA 16802 